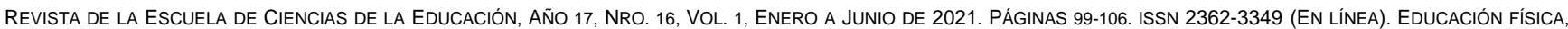
ESCUELA SECUNDARIA Y CLASES MEDIAS. PABLO KOPELOVICH. ALEJO LEVORATTI.

\title{
EDUCACIÓN FÍSICA, ESCUELA SECUNDARIA Y CLASES MEDIAS
}

\author{
Pablo Kopelovich* \\ CONICET, Universidad Nacional de Rosario, Argentina \\ kopelovichp@gmail.com \\ Alejo Levoratti*
CONICET, Universidad Nacional de Rosario, Argentina
levoratti@gmail.com
}

Recibido: 16/07/2020 - Aceptado: 14/10/2020

Resumen

Se justifica la realización de este trabajo para aportar al debate sobre los sentidos que la Educación Física tiene en la escuela media y el imaginario social que existe entre está y la pertenencia de clase de los estudiantes. El objetivo es indagar en los sentidos que adquiere la asignatura en una institución secundaria de la provincia de Buenos Aires destinada a sectores sociales medios-altos, focalizando en las narrativas de profesores/as, en observaciones de clases, y en programas de esta asignatura. Metodológicamente, se realiza desde un enfoque no estándar de investigación. En base a lo expuesto se cumplimentó un estudio de caso en un establecimiento educativo de nivel medio de gestión privada. Los resultados son que las perspectivas de los profesores sobre la Educación Física están atravesadas fuertemente por el deporte, construyéndose cuatro grandes sentidos en torno a esta, vinculados a la transmisión de valores, la creación de hábitos saludables, a la generación de diversión y a los lineamientos del Diseños Curriculares para la provincia de Buenos Aires. Se concluye que las ponderaciones que realizaron los profesores de Educación Física sobre la disciplina se encuentran influenciados por las trayectorias conceptuales de las instituciones donde se formaron, las concepciones imperantes en los Diseños Curriculares jurisdiccionales, los discursos considerados como legítimos en la disciplina, la propuesta curricular disciplinar de las instituciones y los lineamientos que direccionan su propuesta educativa institucional.

Palabras clave: Deporte - Educación - Distinción - Sentidos - Clases medias - Escuela Secundaria.

\section{PHYSICAL EDUCATION, HIGH SCHOOL AND MIDDLE CLASSES}

\begin{abstract}
The realization of this work is justified in its inclusion in the debate on the particularity in the senses of Physical Education at school, based on the social imaginary about the class membership of the students. The objective is to investigate in the senses that the subject acquires in a secondary institution in the province of Buenos Aires destined for upper-middle social sectors, focusing on the narratives of teachers, in class observations, and in programs of this

\footnotetext{
*Profesor y Licenciado en Educación Física (UNLP). Magíster en Ciencias Sociales con orientación en Educación (Facultad Latinoamericana de Ciencias Sociales). Doctorando en Ciencias de la Educación (UNLP). Becario doctoral CONICET. Instituto de Investigaciones en Humanidades y Ciencias Sociales- Universidad Nacional de La Plata (IdlHCS-unlp); y Consejo Nacional de Investigaciones Científicas y Técnicas (CONICET). Docente de la Universidad Nacional de La Plata.

Sus temas de investigación son: la Educación Física escolar, la enseñanza del fútbol, la Historia de la Educación Física en clave de género.

* Doctor con Mención en Ciencias Sociales y Humanas (UNQ). Magíster en Antropología Social (UNSAM). Profesor y Licenciado en Educación Física (UNLP). Investigador del CONICET/IdIHCS-UNLP y Docente de la Universidad Nacional de La Plata. Sus temas de investigación son: El deporte y la educación física en políticas públicas, estudios antropológicos sobre/en el deporte y los procesos de formación y configuración profesional de los profesores de educación física.
} 
subject. Methodologically, it is carried out from a non-standard research approach. Based on the above, a case study was completed in a privately managed medium-level educational establishment. The results are that the teachers' perspectives on Physical Education are strongly crossed by sport, building four great senses around it, linked to the transmission of values, the creation of healthy habits, the generation of fun and guidelines of the Curricular Designs for the province of Buenos Aires. It is concluded that the weightings made by the Physical Education teachers on the discipline are informed by the conceptual trajectories of the institutions where they were formed, the prevailing conceptions in the Jurisdictional Curricular Designs, the discourses considered legitimate in the discipline, the curricular proposal discipline of the institutions and the guidelines that guide their institutional educational proposal.

Keywords: Sports - Education - Distinction - Senses - Middle classes - High School.

\section{Introducción y estado de la cuestión}

Este trabajo se inscribe en una investigación que analizó las representaciones sobre la Educación Física (EF) en una escuela secundaria privada destinada, según los directivos, a clases medias aspiracionales al ascenso social respondiendo parcialmente a aquellas escuelas de las elites que promueven un modelo centrado en un "estilo vincular" (Del Cueto, 2007). En particular, en este artículo se buscará analizar los sentidos asignados a la inscripción de la EF por parte de los profesores de educación física dentro de la propuesta curricular de una escuela destinada, según los directivos, a clases medias, radicada en provincia de Buenos Aires. A partir de este abordaje nos preguntamos: ¿hay una particularidad en los sentidos de la EF en base al imaginario social sobre la pertenencia de clase de los estudiantes en la propuesta curricular y en las narrativas de los profesores de EF? Este interrogante se sustenta en una serie de análisis producidos desde los estudios sociales sobre los deportes donde se analiza cómo las prácticas corporales y en particular algunos deportes en singular operan como elemento de distinción en términos sociales y culturales para determinados grupos sociales (Bourdieu, 1993; luliano, 2010; Fuentes, 2015; Branz, 2015). Además, diferentes abordajes sobre el caso argentino advirtieron cómo los procesos de selección de las prácticas deportivas en las instituciones educativas se relacionan con los imaginarios en términos de clase que tienen los actores institucionales como así también los padres y alumnos. En esos procesos los autores muestran cómo la práctica del rugby entre los varones y el hockey entre las mujeres se vinculan con los sectores sociales altos, operando como un diacrítico de distinción y de construcción de masculinidades y femineidades (Ron, 1995; Uliana, 2010; Fuentes, 2015; Branz, 2015) ${ }^{1}$. En base a ello, es que profundizaremos en los procesos de selección de las prácticas corporales dentro de la propuesta de EF escolar como así también en los sentidos que ponen en circulación los profesores y directivos sobre esta disciplina escolar.

La escuela secundaria, en Argentina y en el mundo, nació con un rol de formadora de elites, a partir de un modelo institucional de carácter selectivo. La referencia de este nivel era el colegio nacional, que preparaba a los alumnos para ingresar a la Universidad y ejercer puestos burocráticos y dirigenciales. Así, la masificación de la escuela media en la Argentina se produjo desde una matriz de incorporación y expulsión en forma simultánea (Acosta, 2012). De esta forma, se pensaba al sistema educativo como un dispositivo de distribución social de valores y conocimientos, a partir del cual cada uno recibía la educación necesaria por el lugar que ocupara en la estructura social (Tedesco, 2012).

En los últimos años, en nuestro país y en la región, el nivel secundario muestra una amplia expansión, transformándose en una cuestión central de las agendas de políticos y expertos (Tiramonti y Montes, 2009). De hecho, en 2006 a través de la Ley de Educación Nacional en Argentina se establece la obligatoriedad del mismo, en concordancia con lo ocurrido en Latinoamérica (Nosiglia, 2007).

Asimismo, en relación especialmente a este nivel presenciamos, en la actualidad, una importante diferenciación entre las distintas instituciones educativas. Esto se produce en un contexto de fragmentación del sistema educativo argentino (Kessler, 2002; Tiramonti 2004; Tiramonti y Ziegler, 2008) y la consiguiente conformación de espacios segregados para la socialización de diferentes grupos, con una diferenciación institucional pronunciada. De esta manera, desde el ámbito académico, se puso la mirada en la educación de la elites o sectores privilegiados de la sociedad, analizando patrones de socialización, como la disciplina escolar y las acciones solidarias (Tiramonti y Ziegler, 2008; Di Piero, 2013; Rodríguez Moyano, 2013; Gessaghi, 2016). Además, se analizó lo que se denominó, a partir de las palabras de los actores sociales estudiados, la educación de la clase alta (Gessaghi, 2016). En ese marco, donde se percibía también una configuración fragmentada de la sociedad, no faltaron las indagaciones en torno a las estrategias educativas de familias residentes en countries y barrios cerrados (Del Cueto, 2007), o a la polarización sociourbana y a la segregación educativa (Veleda, 2005). Asimismo, con respecto a la educación de los sectores privilegiados, se destacaron las investigaciones sobre los exámenes internacionales ofrecidos por ciertas instituciones, como una expresión de modelos emergentes de intervención sobre las instituciones y el trabajo de los profesores que modifican las formas de ordenamiento, control y regulación que habitualmente tuvieron los sistemas educativos modernos (Ziegler, 2011).

Los estudios que analizan la incorporación de la EF en el nivel secundario en la Argentina han enfatizado el carácter deportivista de esta oferta formativa, el cual se inicia a partir de la década de 1940 (Aisenstein, Ganz y Perczyk; 2001; Gómez, 2009). Se ha pensado, además, en las tensiones o contradicciones entre los universos simbólicos del deporte y de la escuela (Bracht y Caparróz, 2009), hablándose en ese contexto de "deporte de la escuela", para referir a un deporte que ha pasado por un proceso de transposición didáctica (tornar a los saberes en saberes escolarizados), y del "deporte en la escuela", para aludir a una práctica no reelaborada.

${ }^{1}$ Un análisis profundo de la cuestión de género para este caso concreto, fue realizado en Kopelovich (2019). 


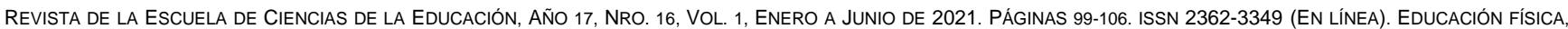
ESCUELA SECUNDARIA Y CLASES MEDIAS. PABLO KOPELOVICH. ALEJO LEVORATTI.

También, una serie de producciones refirieron a una posible "deportivización" del currículum de la EF, y a la condición del deporte como contenido hegemónico (Gómez, 2009). Sin embargo, otro grupo de autores (Aisenstein, Ganz y Perczyk, 2001; Hernández y Carballo, 2002) estima que existe una distancia significativa entre el deporte al que la academia denomina "moderno" (y las asociaciones deportivas y los medios masivos identifican como tal) y el contenido deportivo que se enseña en las clases de EF en la escuela media, tratándose de dos elementos distintos.

Íntimamente vinculado a esta cuestión se encuentra el tema del status de la asignatura. Sáenz y otros (2004) realizan un exhaustivo repaso de los estudios efectuados al respecto. Así, siguiendo a David Kirk (1990), plantean que, para muchos profesores, alumnos y padres, ocupa un estatus educativo bajo. Investigaciones realizadas en distintos países como las de Bain (1990), Schempp (1993), Templin et al. (1994) o Smyth (1995) confirman la marginalidad de esta asignatura en las escuelas, infravalorada por alumnos, colegas y administración. Carr (1983) defiende el estatus educativo de la asignatura porque considera que, por una parte, las actividades físicas implican conocimiento y por otra, reflejan y transmiten cultura. Para el caso de Argentina y Brasil, esta problemática se ha pensado en torno a la idea de identidad y crisis de la EF (Bracht y Crisorio, 2003), presentándose el problema de la identidad en muchas y diferentes dimensiones y prácticas: la constitución del campo de la EF, la legitimación de la misma, el proceso de formación profesional, y la práctica en la institución escolar.

Como podemos observar en este recorrido por la bibliografía sobre la temática, pensar a la educación secundaria implica considerar un proceso de fragmentación y de carriles desiguales que son posibles transitar a partir de los capitales sociales, culturales, económicos de cada familia. Esto lleva a pensar cómo estas tramas institucionales repercutieron en los sentidos sobre la EF y el deporte, posibilitándonos discutir el lugar de los marcos institucionales en los procesos de construcción de sentido sobre la EF y deporte.

En este contexto, cuando nos referimos a los sentidos atribuidos a la asignatura, nos preguntamos por aquellas narrativas que les permiten a los profesores de EF significar su accionar cotidiano en la institución educativa y reconocerse como tales. Es decir, abordamos la dimensión subjetiva de la enseñanza de esta materia, en las concepciones y formas de entender su labor por parte de los docentes.

\section{Metodología}

Los principios metodológicos que direccionan este trabajo nos inscriben dentro de los enfoques no estándares, los cuales buscan comprender interpretativamente las perspectivas de los actores sociales estudiados (Marradi, Archenti y Piovani, 2007). Este enfoque de trabajo nos plantea una reflexión y control permanente entre las problemáticas que direccionan la labor y los datos producidos durante el trabajo de campo, lo cual nos posiciona en diálogo con los diseños de investigación flexibles (Mendizábal, 2006). Para ello, haremos uso principalmente de las técnicas de observación con participación y de entrevistas en profundidad.

En base a lo expuesto se cumplimentó un estudio de caso en un establecimiento educativo de nivel medio de gestión privada. Se realizaron veinte (20) observaciones con participación en las clases de EF como así también en sus instancias previas y posteriores. Las observaciones se cumplimentaron en el primer cuatrimestre de clases del ciclo lectivo de 2015. También, durante dicho período, se efectuaron entrevistas con carácter semi-estructurado a los directivos del establecimiento y a los profesores de EF. Además, se establecieron a lo largo del trabajo de campo conversaciones informales con directivos y profesores de la especialidad.

Por último, se realizó un análisis de los principales documentos institucionales que direccionaban la propuesta educativa, "Acuerdo Institucional de Convivencia" y la propuesta curricular institucional para el área de EF, como así también el material escrito empleado en las diferentes clases.

La unidad de análisis es el nivel secundario del Colegio Kennedy², de gestión privada, de la provincia de Buenos Aires, especialmente los profesores de EF de la misma. Se trata de una escuela laica, mixta, no subvencionada, ubicada en una zona céntrica de la ciudad, con casi cincuenta años de existencia, que cuenta con más de 300 alumnos y cerca de 90 docentes. Se seleccionó esta escuela porque, dentro de las instituciones a las que podíamos tener acceso, resultó ser la que -desde el punto de vista de los directivos- recibía a alumnos de clases medias.

El ingreso al campo se realizó gracias a un contacto con los dueños de la institución educativa por parte de un contacto de uno de los autores. Ello, no obstante, no significó la ausencia de "negociar" permanentemente el rol del investigador durante el desarrollo de la investigación con los diferentes actores sociales que hacen a la institución.

\section{Resultados}

Hallamos que la Educación Física en el Colegio Kennedy se encontraba fuertemente deportivizada. Afirmamos esto ya que el Director del Departamento de EF al ser consultado sobre la forma en la que se organizaba la enseñanza de la asignatura, planteó que se abordaba un deporte por trimestre. Asimismo, los programas de las materias nos mostraban que prácticamente todos los contenidos incluidos se vinculan directamente a esta práctica cultural. Así, en el programa de $1^{\circ}$ y $2^{\circ}$ año se planteaba para cada período lo siguiente:

Primer Trimestre-Unidad 1: Vóley-Hándbal-Atletismo -Formación Física básica

Segundo Trimestre- Unidad 2: Vóley-Hándbal- Natación- Atletismo-Formación Física básica

${ }^{2}$ El nombre de la institución ha sido modificado para reservar el anonimato de los actores que participaron del proceso de investigación. Asimismo, el nombre original hace referencia a otro presidente de los Estados Unidos perteneciente al partido republicano. 
REVISTA dE LA ESCUELA DE CIENCIAS DE LA EdUCACIÓN, AÑO 17, NRO. 16, VOL. 1, ENERO A JUNIO dE 2021. PÁGINAS 99-106. ISSN 2362-3349 (EN LíNEA). EdUCACIÓN FíSICA, ESCUELA SECUNDARIA Y CLASES MEDIAS. PABLO KOPELOVICH. ALEJO LEVORATTI.

Tercer Trimestre-Unidad 3: Vóley- Hándbal- Natación- Atletismo- Fútbol -Formación Física básica

En lo que respecta al Programa de EF de $3^{\circ}$ y $4^{\circ}$ año y $4^{\circ}$ y $5^{\circ}$ año (respectivamente), se puede leer:

Primer Trimestre- Unidad 1: Voley- Hándbal-Softbol- -Formación Física básica

Segundo Trimestre-Unidad 2: Voley- Hándbal-Softbol-Natacion -Formación Física básica

Tercer Trimestre-Unidad 3: Voley- Hándbal-Sóftbol-Natación - Fútbol- Formación Física básica

Por el lado de las prácticas observadas, efectivamente mostraban que lo que se enseñaba en el marco de esta materia casi no incluía otros saberes: se identificaron contenidos vinculados a la gimnasia solamente en contadas ocasiones y en el acondicionamiento previo. En lo que respecta a la bibliografía obligatoria para los/as estudiantes, se seguía la misma línea al presentar textos relativos a cuestiones técnicas, tácticas, estratégicas y reglamentarias de los deportes mencionados. En este contexto, fue posible identificar cuatro sentidos principales sobre la EF -atravesados claramente por el deporte- en la propuesta del Colegio Kennedy:

\section{a) La transmisión de valores desde la Educación Física}

Se entendía a la EF como una asignatura formadora de buenas personas, transmitiendo valores, mejorando el vínculo entre los alumnos. Así, el profesor 1, al ser consultado sobre cómo le gustaría que los alumnos recuerden sus clases, planteaba que busca "que se acuerden de principios, de valores, de decir la verdad, de ese tipo de cuestiones". En la misma línea, aunque refiriendo al trato entre las personas, el profesor 2 expresó que aspiraba a que "les quede una sensación de que atravesaron un proceso educativo donde el compañero era lo esencial, donde utilizábamos el conocimiento como algo que nos completaba como ser humano, pero era muy importante este tema de tener en cuenta y de aplicar una sensibilidad particular respecto del ser humano que tenemos al lado, que en definitiva es lo que nos permite desarrollarnos como seres sociales".

Entonces, los profesores de la materia entrevistados consideraron que estas cuestiones se producían a partir de la enseñanza de ciertos elementos que atraviesan a todas las materias. De esta forma, explicaron que tenían "como una referencia fundamental, o como contenido transversal, la tolerancia, la ayuda mutua, el respeto por el prójimo, la equiparación del poder que tienen ellos de elegir" (profesor 2). Se aclaró que los contenidos enseñados en clase se abordaban de la mano de los mencionados anteriormente: "Por supuesto que los contenidos conceptuales se desarrollan: los chicos aprenden sóftbol, los chicos aprenden vóley, atletismo, pero en todo eso es muy fuerte la filosofía esta de la tolerancia mutua, del respeto" (profesor 2); "Hay contenidos puntuales de la asignatura y hay principios que son de vida. Van congeniados los dos. Trato de que en mi clase el pibe no mienta, no insulte, diga la verdad, trabaje, entienda que yo no le miento" (profesor 1). Aquí es interesante cómo, por un lado, se enseñaba lo que los actores denominaron "contenidos de la asignatura" y, por el otro, se transmitieron los valores y los principios de vida. Una serie de situaciones en las clases observadas nos permite pensar que se generaron momentos de reflexión sobre el "buen comportamiento", el trato respetuoso entre las personas, y el compañerismo.

No obstante, esas situaciones no se dieron en circunstancias vinculadas estrictamente con las clases de EF, lo que apoyaría lo referido anteriormente en relación a pensar contenidos y valores por separado. Es decir, no se observó que a través del deporte se transmitieran valores como la ayuda mutua, el respeto, el compañerismo. Sin embargo, solamente un profesor (profesor 4) -al ser consultado sobre la evaluación- hizo referencia a la transmisión de valores -en este caso el compañerismo- a través del deporte: "Me interesa que estén comprometidos porque saben que, si uno no está listo para jugar, el equipo juega con uno menos. Rescato que ahora [jugando al fútbol] están todos atentos, no están mirando a las chicas o por ahí".

Hallamos que este sentido en torno a la EF en el colegio marca una continuidad con el Proyecto de la institución. Así, desde los documentos institucionales se insistía en la idea de transmitir valores. De esta forma, se decía que la familia debía "apoyar la gestión institucional en el marco de una convivencia caracterizada por los valores compartidos" (Acuerdo Institucional de Convivencia, 2015, p.11); se hacía referencia a "un marco de sólidos valores individuales y democráticos" (Acuerdo Institucional de Convivencia, 2015, p.13), de "poner en práctica los valores que posibilitan y promueven la convivencia armónica y democrática" (Acuerdo Institucional de Convivencia, 2015, p.13), entre otras cosas.

En este contexto, la vicedirectora planteó que "el nombre del Colegio [Kennedy] tiene que ver con estos valores que para la escuela son esenciales, como es la solidaridad, el respeto, la diversidad, al otro. El valor de la libertad es esencial y, entonces, formamos a nuestros jóvenes en esos pilares esenciales". Asimismo, el director aludía a respeto, responsabilidad y compromiso.

\section{b) La promoción de hábito saludables y de prácticas deportivas extraescolares}

Se trata de una concepción sobre la disciplina que presenta distintas aristas. Entre estas, se encontró la de verla como creadora de hábitos de vida saludables (realización de ejercicio físico), como generadora de interés en los alumnos por las prácticas corporales y como forma de acercar a estos a algún club para realizar prácticas deportivas, entre otras. Es decir, de ser útil para la vida cotidiana de los estudiantes. Se trata, en realidad, de una serie de significados íntimamente vinculados entre sí, que tienen en común la idea de pensar a la asignatura más allá del espacio y tiempo de la clase y de la institución escolar.

Así, el profesor 2 al ser consultado sobre los contenidos que priorizaba a la hora de enseñar la materia, expresó que buscaba que "(...) el contenido sea aplicable a la vida cotidiana en general, no al espacio de la escuela", opinando, además, que en la institución "se presta mucha atención a que esos conocimientos conceptuales pueden ser utilizados por los alumnos más allá de nuestra presencia. Yo creo que es algo que atraviesa, o nos atraviesa, a todos los docentes". 


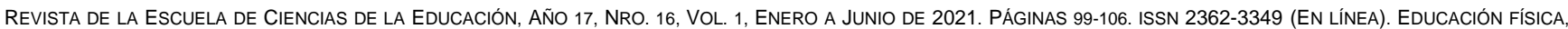
ESCUELA SECUNDARIA Y CLASES MEDIAS. PABLO KOPELOVICH. ALEJO LEVORATTI.

También, se piensa en este Colegio a la asignatura vinculada a fomentar la práctica deportiva en los clubes. Esto puede verse en varios pasajes de las entrevistas realizadas. Una profesora al ser consultada sobre lo que puede aportar la EF a la educación de los jóvenes, respondió: "una predisposición para [practicar] deportes afuera, si quieren seguir algún deporte extraescolar" (profesora 1) ${ }^{3}$; mientras que otro docente, ante la misma pregunta, plantea que "les damos el puntapié inicial para que hagan un deporte" (profesor 4).

En relación a la promoción de hábitos de vida saludable, el profesor 1 lo entendía de la siguiente manera: "tiene que ver con el estilo de vida, con salud, con beneficios, que te da la actividad física".

Todas las anteriores ideas desarrolladas en torno a la EF circulan en un contexto en el que los docentes consultados consideraban que, en líneas generales, los alumnos eran más sedentarios y "menos hábiles" motrizmente en comparación con los jóvenes del pasado. Entonces, la EF estaría siendo pensada como medio para enfrentar el sedentarismo que presentarían los alumnos.

\section{c) Educación Física generando diversión o disfrute}

Un tercer sentido atribuido a la asignatura tiene que ver con entenderla como una práctica que busca generar en los alumnos diversión o disfrute. Vale aclarar que esta forma de ver a la materia en cuestión surgió, especialmente, cuando se los consultó a los profesores sobre lo siguiente: ¿Qué es lo que más les gusta a los alumnos de tus clases o de la EF de este colegio en general? Y ¿Qué crees que consideran los alumnos en relación a la asignatura en esta escuela?

Con respecto a qué es lo que más les gusta a los alumnos de las clases de EF, en algunos casos los profesores plantearon que lo que preferían, especialmente, era jugar al fútbol. En relación a esto, uno de los profesores de $6^{\circ}$ año les explicaba a los alumnos que, si se trabaja bien durante todo el año, finalizando el ciclo lectivo podían dedicarle unas clases a la enseñanza de este deporte. En uno de los casos en los que se mencionaba al fútbol, el docente agregó que "en realidad disfrutan (...) de todos los deportes, siempre y cuando puedan jugar un rato, más allá de que se puedan practicar las cuestiones técnicas, siempre se juega. Intentamos que gran parte de la clase sea juego" (profesor 3). En otra ocasión, se fue en la misma línea al considerar que "es como que ellas creen que vienen a jugar y se distraen un poco de lo que hacen habitualmente en el aula" (profesora 1).

Además, en otro caso, se incorporó el tema de la negociación con los alumnos a partir de esta situación: "Les gusta hacer algo que les guste. Eso les gusta. O sea, si ellos hacen lo que les gusta, está bueno. Entonces, ahí hay que aprender a negociar y tengo que ver cómo lo doy para que a ellos les termine gustando". Podría decirse que aquí se ve el fuerte vínculo que existiría entre la práctica de la EF y el disfrute. Es decir, a partir de las palabras de este docente puede suponerse que para que los alumnos se desempeñen de forma satisfactoria debía necesariamente gustarles lo que hacían.

En relación al interrogante sobre qué consideraban que creían los alumnos sobre la asignatura, es llamativo cómo casi en todas las respuestas, se pensó la cuestión en el mismo sentido que en la pregunta anterior (qué es lo que más les gusta a los alumnos de la clase de EF). Así, nos encontramos con contestaciones como: "Hay pibes que se mueren por ir a Educación Física, hay pibes que les es una carga" (profesor 2); y "lo ven como un espacio de disfrute, más que cualquier otra materia que les pueda gustar" (profesor 1). Es decir, desde la visión de los profesores, los alumnos al pensar en la asignatura reflexionan exclusivamente sobre si les gusta o no les gusta, desatendiendo cuestiones estrictamente pedagógicas como qué les enseñan. Incluso una de las profesoras al ser consultada sobre la segunda pregunta (qué consideran los alumnos en relación a la asignatura) no logra ver la diferencia con respecto a la primera (qué es lo que más les gusta).

\section{d) EI Diseño Curricular en las perspectivas de los profesores}

Este último sentido que adquiría la asignatura en este nivel de esta institución educativa se construye a partir de la lectura de las planificaciones para cada año. Las mismas fueron realizadas agrupando dos años: $1^{\circ}$ y $2^{\circ}$ año, $3^{\circ}$ y $4^{\circ}$ año, y $4^{\circ}$ y $5^{\circ}$ año. Allí encontramos que se retomaron distintos conceptos presentes tanto en el Diseño Curricular de Educación Física para provincia de Buenos Aires para las escuelas convencionales, como así también del Diseño Curricular de la asignatura "Educación Física y Corporeidad", correspondiente al $4^{\circ}$ año de la orientación en EF.

De este modo, en la sección "Contenidos", en todos los casos, se organizó la asignatura a partir de los mismos tres ejes que figuran en el Diseño de Secundaria: Corporeidad y Motricidad, Corporeidad y Sociomotricidad, y Corporeidad y Motricidad en relación con el ambiente. Los que variaron fueron los contenidos que se extrajeron del mismo documento, incluidos en cada uno. En esos contenidos, identificamos elementos propios de la Praxiología Motriz, como, por ejemplo: "El principio de salud como orientador básico para la realización de las tareas motrices" (contenidos de $1^{\circ}$ y $2^{\circ}$ año), y "Las acciones motrices específicas para resolver situaciones de desplazamiento sobre terrenos y accidentes naturales" (contenidos $5^{\circ}$ y $\left.6^{\circ}\right)$.

Además, en los contenidos encontramos alusiones a la búsqueda y el mantenimiento de la salud a partir de la EF: "Los principios de salud, individualización, recuperación, concientización y utilidad. Su consideración en la secuenciación de proyectos personales de formación corporal y motriz" (contenidos de $5^{\circ}$ y $6^{\circ}$ año). También, los siguientes fragmentos de otras secciones van en la misma línea: "Conozcan las actividades motrices necesarias para el desarrollo de las capacidades condicionales y coordinativas con base en principios de salud" (Expectativas de logro de $1^{\circ}$ y $2^{\circ}$ años), "(...) producir sus secuencias personales de prácticas motrices, con base en los principios de salud, individualización, recuperación, utilidad y concientización" (Objetivos de enseñanza de $5^{\circ}$ y $6^{\circ}$ años).

\footnotetext{
${ }^{3}$ Profesora en Educación Física egresada de la Universidad Nacional de La Plata que se desempeña también en el nivel primario de la
} institución educativa en cuestión. 
REVISTA dE LA ESCUELA DE CIENCIAS DE LA EdUCACIÓN, AÑO 17, NRO. 16, VOL. 1, ENERO A JUNIO dE 2021. PÁGINAS 99-106. ISSN 2362-3349 (EN LíNEA). EdUCACIÓN FíSICA, ESCUELA SECUNDARIA Y CLASES MEDIAS. PABLO KOPELOVICH. ALEJO LEVORATTI.

\section{Discusión y conclusiones}

En la presentación de los datos producidos en el trabajo de campo lo primero que observamos fue el proceso de deportivización de las prácticas de la EF escolar. En el caso estudiado no se estableció relación entre la práctica deportiva propuesta y determinadas prácticas de distinción en términos de clase social. De este modo, en un contexto en el que en dicha asignatura ocupa un lugar central la práctica deportiva, la inclusión de deportes como hándbol, vóley, sóftbol, fútbol y atletismo -estimados como tradicionales por las instituciones educativas en general- no tensionaría a favor de aportar distinción, exclusividad, en la socialización de alumnos presuntamente de clases medias. Esto sí sucedería en las escuelas secundarias -como las mencionadas por Del Cueto (2007) a las que concurren familias residentes de countries y barrios cerrados- donde se práctica hockey, rugby, fútbol, golf, equitación y hasta deportes náuticos. Asimismo, la autora plantea que algunos de esos colegios organizan viajes para participar de torneos intercolegiales tanto en el país como el exterior (Del Cueto, 2007, p. 55). Es decir, que, en ese proceso de sumisión de la EF a los deportes en el establecimiento estudiado, no se establecieron particularidades en los sentidos promovidos de acuerdo al deporte como así tampoco se erigieron prácticas singulares en términos de elemento de distinción.

En el desarrollo también se observa la convivencia de múltiples sentidos sobre las prácticas de la EF. Es decir que sería una reducción de esta problemática social buscar la existencia de un sentido único y legítimo para los actores instituciones. Ahora bien, advertimos que las ponderaciones que realizaron los profesores de EF sobre la disciplina se encontraban atravesadas por la trayectoria conceptual de las instituciones donde se formaron, los discursos legítimos en la disciplina, la propuesta curricular disciplinar de la institución, los diseños curriculares provinciales y los lineamientos que direccionaron a la propuesta educativa institucional. En ese complejo entramado se produjeron los sentidos de la EF por parte de los profesores.

Como planteamos, es posible identificar cuatro grandes sentidos en torno a la enseñanza de esta asignatura. El primero de estos referiría a una educación en valores: la práctica deportiva permitiría la adquisición de ciertos valores. Sin embrago, no hemos encontrado esto en las observaciones, en concordancia con Raúl Gómez quien expresó que "la actitud cooperativa, solidaria, la sensibilidad ante la injusticia, la autonomía de pensamiento, el juicio crítico, están potencialmente presentes en las situaciones de la práctica deportiva, pero la investigación sugiere que no se derivan de esta con grado de necesidad" (Gómez, 2009, p.250).

El segundo sentido nos permite pensar en los cambios históricos acaecidos en la escuela que pasa de ser entendida -siguiendo a Dubet (2010)- como un santuario, en el sentido de que debía protegerse de los "desórdenes y pasiones del mundo" a ser considerada como aportando capacidades útiles. Asimismo, es posible identificar la corriente de la EF ligada a la "Salud Integral", destacando los beneficios de la actividad física desde lo fisiológico, lo psicológico y lo social (Sánchez, 1996).

El tercer sentido mostraría la tendencia que ve en la EF una finalidad lúdica, considerando su función catártica como uno de sus principales aportes. En este sentido, las investigaciones de Placek (1983) sobre el pensamiento de los profesores de EF indican que el éxito en su enseñanza reside en "mantener ocupados, felices y contentos a los estudiantes". O sea, que lo más importante para los docentes consistiría en el disfrute y en la participación del alumnado, así como en evitar problemas de comportamiento en las clases, muy por encima de la relevancia concedida al contenido (Devís Devís, Fuentes y Sparkes, 2007).

Finalmente, el último de estos sentidos nos muestra cómo fueron recuperados en la propuesta de esta institución elementos propios de la normativa estatal entre los que se destacan los conceptos de la Praxiología motriz, y las referencias a una EF que busca la Salud Integral (como también planteábamos en el segundo sentido).

En base a ello, llegamos a la discusión de dos cuestiones: la primera está ligada a la posibilidad de pensar un deporte escolar. Ante ello advertimos la imposibilidad de considerar tal práctica sin atender a los actores sociales que producen el fenómeno, es decir, en este caso a los profesores de EF. Esta cuestión, rompe con la idea de un marco institucional escolar que promueva sentidos unívocos como así también la idea de un deporte escolar estructurado simbólicamente solo por la institución. Como se pudo observar la producción de sentidos sobre el deporte la realizaron en las intersecciones de diferentes líneas de significación (Levoratti, 2015). La segunda cuestión que buscamos poner en discusión se relaciona a la posibilidad de pensar las particularidades que tendría la EF inscripta en un establecimiento destinado a las clases medias y la producción de estrategias de distinción. En ese contexto, lo primero que tenemos que hacer es romper con abordajes que piensan de modo lineal tal asociación. En este caso, observamos que los sentidos de la propuesta disciplinar se encontraron referenciados en los imaginarios de clase social, promoviendo el aprendizaje de ellos en la práctica deportiva desarrollada en la clase de EF. Ante ello, entendemos que los criterios de percepción y de acción de los profesores, con sus trayectorias como docentes, y el perfil social institucional direccionado a los sectores universitarios, comerciantes y de servicios con un determinado poder adquisitivo en términos económicos, estructuraron la producción de sentido. Ello permite explicar la particularidad de dos cuestiones. La primera es la diferencia que se presentó con otros establecimientos en la selección de las prácticas corporales donde no se reprodujeron los estereotipos que ligan un deporte a una clase social. Esto se debe a que en este mundo simbólico se ponderaba la realización de deportes, por la producción de valores burgueses, pero no se referenciaba en el ámbito escolar la producción de un diacrítico de distinción a partir de una práctica en particular. Ello no significa que estos actores sociales participen en otros espacios sociales, como los clubes, donde sí se promueva ello. La segunda cuestión está en relación a que en términos materiales y simbólicos no tendría las mismas connotaciones realizar un determinado deporte en diferentes espacios sociales. Es decir, no es lo mismo practicar un deporte en un club o en un establecimiento educativo con una trayectoria en los círculos de sociabilidad de esta práctica que ejecutarlo circunscripto a una clase de Educación Física. En 


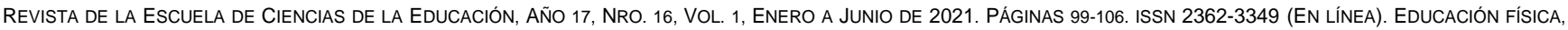
ESCUELA SECUNDARIA Y CLASES MEDIAS. PABLO KOPELOVICH. ALEJO LEVORATTI.

base a ello, podemos afirmar que las prácticas deportivas en la clase de Educación Física escolar tienen relevancia en la formación de este grupo social, pero no es posible reducir ello a un deporte particular.

Este recorrido nos abre dos interrogantes para indagar en próximos trabajos: ¿Cuáles son las valoraciones que realizan los padres sobre la clase de Educación Física y la realización en ella de deportes para la educación de sus hijos? ¿Qué lugar ocupan las prácticas de la EF escolar en la producción cultural de los cuerpos de estas clases sociales”?

\section{Referencias bibliográficas}

Acosta, F. (2012). La escuela secundaria argentina en perspectiva histórica y comparada: modelos institucionales y desgranamiento durante el siglo XX. En: Cadernos de História da Educação, 11(1), pp. 131-144. Disponible en: https://bit.ly/2J8IXdc

Aisenstein, A.; Ganz, N. y Perczyk, J. (2001). El deporte en la escuela. Los límites de la recontextualización. En: Lecturas: Educación Física $y$ deportes, 6(30). Disponible en: https://bit.ly/2Uzd41K

Bain, L. (1990). Physical education teacher education. En: Houston, Handbook of Research on Teacher Education. A projet of the Association of Teacher Educators, pp. 758-782. Macmillan Publishing Company.

Bracht, V. y Crisorio, R. (2003). La Educación Física en Argentina y Brasil. Identidad, desafíos y perspectivas. Argentina: Al Margen.

Bracht, V. y Caparróz, F. (2009). El Deporte como contenido de la Educación Física escolar: la perspectiva crítica de la Educación Física brasileña. Martínez Álvarez, L. y Gómez, R. (coord.), La Educación física y el deporte en la edad escolar, pp. 53-89. Argentina: Miño y Dávila.

Branz, J. (2015). Deporte y masculinidades entre sectores dominantes de la ciudad de La Plata. Estudio sobre identidades, género y clase [Tesis doctoral. Universidad Nacional de La Plata]. Repositorio Institucional UNLP. Disponible en: https://bit.ly/2QQtZeX

Carr, D. (1983). The place of physical education in the school curriculum. En: Momentum, 8(1), pp. 9-12.

Del Cueto, C. (2007). Los únicos privilegiados. Estrategias educativas de familias residentes en countries y barrios privados. Buenos Aires: Prometeo Libros.

Devís Devís, J.; Fuentes Miguel, J. y Sparkes, A. (2006). ¿Qué permanece oculto del currículum oculto? Las identidades de género y de sexualidad en la Educación Física. En: Revista Iberoamericana de Educación, 39, pp. 73-90. Disponible en: https://bit.ly/2WDoh3M

Di Piero, E. (2013). Tensiones entre la inclusión y la selección en la escuela media: el caso de un grupo de escuelas tradicionales de la ciudad de La Plata [Ponencia en Jornada]. 2da Reunión internacional sobre formación de las elites: enfoques y avances de investigación en el estudio relacional de las desigualdades, Buenos Aires, Argentina. Disponible en: https://bit.ly/2QIB94T

Dubet, F. (2010). Crisis de la transmisión y declive de la institución. En: Política y Sociedad, 47(2), pp. 15-25. Disponible en: https://bit.ly/3dsJGms

Fuentes, S. (2015). La formación moral de los jóvenes de elite en circuitos de educación privada en Buenos Aires. En: Pro-Posições, 26(2), pp. 75-98. Disponible en: https://bit.ly/3dq9L5n

Gessaghi, V. (2016). La educación de la clase alta en Argentina. Entre la herencia y el mérito. Argentina: Siglo XXI.

Gómez, R. (2009). Pedagogía del deporte y reflexividad: elementos para una teoría de la enseñanza del deporte en la escuela latinoamericana, en: Martínez Álvarez, L. y Gómez, R. (coord.). La Educación física y el deporte en la edad escolar. Argentina: Miño y Dávila.

Hernández, N. y Carballo, C. (2002). Acerca del concepto de deporte. Alcance de su(s) significado(s). En: Revista de Educación Física y Ciencia, 6, pp. 87-102. Disponible en: https://bit.ly/2WIWkYA

Iuliano, R. (2010). Ocio, consumo y deporte entre los estratos superiores: Aportes para la elaboración de un campo problemático. En: Revista Educación Física y Ciencia, 12, pp. 39-54. Disponible en: https://bit.ly/3agGM29

Kessler, G. (2002). La experiencia escolar fragmentada. Estudiantes y docentes en la escuela media en Buenos Aires. Buenos Aires: IIPEUNESCO.

Kirk, D. (1990). Educación Física y Currículum. España: Universitat de Valencia.

Kopelovich, P. (2019). Fútbol como práctica de exaltación de masculinidades. El caso de un colegio secundario de sectores medios de la provincia de Buenos Aires, Argentina. En: Cuadernos, 55, pp. 65-84. Disponible en: http://revista.fhycs.unju.edu.ar/revistacuadernos/index.php/cuadernos/article/view/318

Levoratti, A. (2015). Deporte y política socio-educativa. Buenos Aires: Prometeo Libros.

Marradi, A.; Archenti, N. y Piovani, J. I. (2007). Metodología de las ciencias sociales. Buenos Aires: Emecé.

Mendizábal, N. (2006). Los componentes del diseño flexible en la investigación cualitativa, en: Vasilachis, I. (coord) Estrategias de investigación cualitativa. España: Gedisa.

Nosiglia, M. (2007). El proceso de sanción y el contenido de la Ley de Educación Nacional №26206: continuidades y rupturas. En: Práxis educativa, 11, pp. 113-138. Disponible en: https://bit.ly/2xjNLbW

Parlebas, P. (1981). Contribution à un lexique commenté en science de l'action motrice [Ponencia]. IV Seminario internacional actividad física y deporte. INEFC-Centre de Barcelona, España.

Placek, J. H. (1983). Conceptions of Success in Teaching: Busy, Happy and Good? en; Templin, T. J. y Olson, J.K (eds.). Teaching in Physical Education, pp. 46-56. Human Kinetics.

Rodríguez Moyano, R. (2013). Elite social, ¿elite educativa? Experiencias escolares en escuelas privilegiadas de Buenos Aires [Ponencia]. 2da Reunión internacional sobre formación de las elites: enfoques y avances de investigación en el estudio relacional de las desigualdades, Buenos Aires, Argentina. Disponible en: https://bit.ly/3apBfGq

Ron, O. (1995). Experiencia en rugby escolar en una escuela privada de Ranelagh. En: Serie Pedagógica, 2, pp. 51-64.

Sáenz-López Buñuel, et al. (2004). El estatus de la Educación Física desde el punto de vista de la opinión de su profesorado. En: Revista Lecturas: Educación Física y deportes, 10, №70, pp. 1-4. Disponible en: https://bit.ly/2Uu5QMm

Sánchez Bañuelos, F. (1996). La actividad física orientada a la salud. Argentina: Biblioteca Nueva.

Schempp, P.G. (1993). Constructing Professional Knowledge: A Case Study of an Experienced High School Teacher. En: Journal of Teaching in Physical Education, 13(1), pp. 3-25. Disponible en: https://doi.org/10.1123/jtpe.13.1.2

Smyth, D.M. (1995), First-Year Physical Education Teachers' Perceptions of Their Workplace. En: Journal of Teaching in Physical Education, 14(2), pp. 198-214. Disponible en: https://doi.org/10.1123/jtpe.14.2.198

Tedesco, J. C. (2012). Educación y justicia social en América Latina. Argentina: Fondo de Cultura Económico.

Templin, T.J., et al. (1994). Matching the Self: the Paradoxical Case and Life History of a Late Career Teacher/Coach. En: Journal of Teaching in Physical Education, 13(3), pp. 274-294. Disponible en: https://doi.org/10.1123/jtpe.13.3.274

Tiramonti, G. (2004). La trama de la desigualdad educativa: mutaciones en la escuela media. Argentina: Manantial.

Tiramonti, G. y Ziegler, S. (2008). La educación de las elites. Argentina: Paidós. 


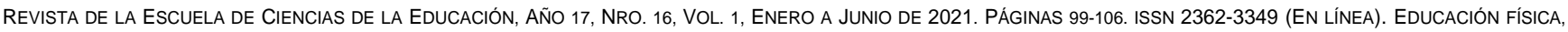
ESCUELA SECUNDARIA Y CLASES MEDIAS. PABLO KOPELOVICH. ALEJO LEVORATTI.

Tiramonti, G. y Montes, N. (2009). La escuela media en debate. Problemas actuales y perspectivas de la investigación. Buenos Aires: Manantial/FLACSO.

Veleda, C. (2005). Efectos segregatorios de la oferta educativa. Documento de Trabajo CIPPEC, 5, pp. 1-62. Disponible en: https://bit.ly/2QF9phO

Ziegler, S. (2011). La era de los exámenes: Bachillerato Internacional, regulaciones posburocráticas y trabajo docente. En: Propuesta Educativa, 36(2), pp. 45-57. Disponible en: https://bit.ly/2wnBVO1 